\title{
Inhibition of poly (ADP-ribose) polymerase and inducible nitric oxide synthase protects against ischemic myocardial damage by reduction of apoptosis
}

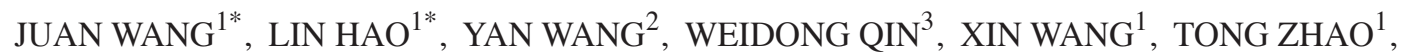 \\ YUSHENG LIU $^{1}$, LIN SHENG ${ }^{1}$, YIMENG DU ${ }^{1}$, MENGYUAN ZHANG $^{2 *}$ and QINGHUA LU $^{1 *}$ \\ ${ }^{1}$ Department of Cardiovascular Medicine, The Second Hospital of Shandong University, Jinan, Shandong 250033; \\ ${ }^{2}$ Department of Anesthesiology, Provincial Hospital Affiliated to Shandong University, Jinan, Shandong 250062; \\ ${ }^{3}$ Department of Intensive Care Unit, Qilu Hospital Affiliated to Shandong University, \\ Jinan, Shandong 250012, P.R. China
}

Received February 9, 2014; Accepted September 19, 2014

DOI: $10.3892 / \mathrm{mmr} .2014 .2977$

\begin{abstract}
Myocardial infarction (MI) is defined as the deprivation of the myocardial tissue of oxygen and nutrients, resulting in the induction of inflammation and apoptosis of the cardiomyocytes. Poly (ADP-ribose) polymerase 1 (PARP1) is a nuclear enzyme closely associated with MI, that can be activated by DNA damage. Inducible nitric oxide synthase (iNOS) is a critical enzyme among the inflammatory cytokines. The present study aimed to investigate the underlying mechanism of the protective effects of PARP1 and iNOS inhibitor against $\mathrm{MI}$, in rats. A total of 40 male Wistar rats were divided into four groups. The rats were anesthetized with sodium pentobarbital $(50 \mathrm{mg} / \mathrm{kg})$, and the left anterior descending coronary artery was occluded by ligation, using a 6-0 polypropylene monofilament suture, at the left atrial apex, in order to induce MI. The rats from each group received an abdominal injection of either dimethylsulfoxide (100 $\mu 1$, for MI group); PARP-1 inhibitor, 3,4-dihydro-5-[4-(1-piperidinyl)butoxy]-1(2H)isoquinolinone (DPQ; $10 \mathrm{mg} / \mathrm{kg}$ ); or iNOS inhibitor, $\mathrm{N}$-(1-naphthyl)ethylenediamine dihydrochloride (1400W; $10 \mathrm{mg} / \mathrm{kg}$ ). The hearts were harvested from the rats after four weeks. Inhibition of PARP and iNOS activity improved heart function, as determined by serial echocardiography. The
\end{abstract}

Correspondence to: Dr Qinghua Lu, Department of Cardiovascular Medicine, The Second Hospital of Shandong University, 247 Bei Yuan Street, Jinan, Shandong 250033, P.R. China

E-mail: lqyzhy@163.com

Dr Mengyuan Zhang, Department of Anesthesiology, Provincial Hospital Affiliated to Shandong University, 81 Jingshi Road, Jinan, Shandong 250062, P.R. China

E-mail: zhangmy717@163.com

*Contributed equally

Key words: myocardial infarction, poly (ADP-ribose) polymerase 1, inducible nitric oxide synthase, apoptosis, peroxynitrite rate of apoptosis, as determined by a terminal deoxynucleotidyl-transferase-mediated dUTP nick end labeling assay, was reduced by 39.71 and $39.00 \%$ in the DPQ and 1400W groups, respectively, and this was accompanied by the downregulated expression of cleaved caspase-3 and PARP1. Effective inhibition of PARP and iNOS, by DPQ and $1400 \mathrm{~W}$, was detected by western blotting and immunofluorescence, and was shown to repress $\mathrm{O}_{2}{ }^{-}$and nitrotyrosine levels, following MI. The present study confirmed that inhibition of PARP1 and iNOS was able to protect against ischemic myocardial damage, by reducing the levels of apoptosis.

\section{Introduction}

Myocardial infarction (MI) is a common cardiovascular disease, and one of the leading causes of mortality, worldwide (1). MI is caused by myocardial deprivation of oxygen and nutrients, which results in the induction of the inflammatory response and apoptosis of cardiomyocytes (2). Various factors are involved in the process, including poly (ADP-ribose) polymerase 1 (PARP1).

PARP1 is a nuclear enzyme, which functions as a DNA damage sensor, and can be activated by DNA strand breaks (3). Activation of PARP1 leads to the synthesis of poly(ADP-ribose) (PAR), from nicotinamide adenine dinucleotide (NAD) and ATP, which is essential for DNA repair (4). However, excessive activation of PARP1 may result in the depletion of NAD and ATP, which leads to cellular dysfunction and eventual cell death (5). Furthermore, PARP1 is required for specific $\mathrm{NF}-\kappa \mathrm{B}$-dependent gene activation, and acts as a transcriptional co-activator of $\mathrm{NF}-\kappa \mathrm{B}(6)$. It has been shown to regulate various key inflammatory cytokines, including monocyte chemotactic protein-1, inducible nitric oxide synthase (iNOS), and adhesion molecules, all of which are known to be regulated by $\mathrm{NF}-\kappa \mathrm{B}(7-9)$. Excessive activation of PARP1 has been shown to be associated with the pathogenesis of numerous diseases, including energetic failure and vascular collapse in shock, diabetes, cerebral ischemia, endothelial dysfunction in hypertension, atherosclerosis, and 
heart failure (10-12). Martinet et al (13) previously provided evidence of elevated levels of PARP1 in human atherosclerotic plaques. In addition, PARP1 inhibition has been demonstrated to provide protection against endothelial dysfunction in shock, hypertension, and heart failure (14).

iNOS is a critical member among the inflammatory cytokines regulated by PARP1 through the NF- $\mathrm{B}$ pathway. Induction of iNOS results in excessive production of nitric oxide (NO), which reacts with superoxide anions to form peroxynitrite. The production of peroxynitrite results in tyrosine nitration, DNA damage, and activation of PARP1, which leads to changes in inflammatory responses, and promotion of cell death by apoptosis and necrosis $(11,15)$. Numerous studies have demonstrated that neutralization of peroxynitrite is an effective therapeutic against cardiovascular, inflammatory, and neurodegenerative diseases, by providing protection against cell death and downregulating inflammatory responses (15).

In the present study, it was hypothesized that the oxidative DNA damage, that results from the generation of reactive species during the onset of MI, may cause excessive activation of PARP1. Excessive PARP1 may result in an increased expression of iNOS, and an imbalance of cell survival mechanisms that contribute to the death of cardiomyocytes and aggravation of cardiac functions. It may be hypothesized that pharmacological inhibition of PARP1 or iNOS may protect cardiomyocytes from death, and improve cardiac function. A rat model of MI was used to investigate the potential role of PARP1 and iNOS in the process of MI, and to examine the protective effects of their inhibition.

\section{Materials and methods}

Animals and surgery. A total of 40 male Wistar rats (Animal Experiment Center of Shandong University, Jinan, China), 4 months old, were housed and bred in a pathogen-free animal care facility at the Key Laboratory of Cardiovascular Remodeling and Function Research (Jinan, China). All of the rats were allowed full access to standard mouse chow and water. All experiments were performed in compliance with the Guide for the Care and Use of Laboratory Animals, published by the US National Institutes of Health (NIH Publication No. 85-23, revised 1985; NIH, Bethesda, MA, USA) and Shandong University (Jinan, China).

The rats were anesthetized for a sham operation with sodium pentobarbital $(50 \mathrm{mg} / \mathrm{kg})$. In order to induce an $\mathrm{MI}$, the left side of the chest was opened and the left anterior descending coronary artery was occluded by ligation, using a 6-0 polypropylene monofilament suture at the left atrial apex, as described by previous methods (16). Immediately following coronary ligation, all of the rats received a single abdominal injection of either dimethylsulfoxide (DMSO; $100 \mu \mathrm{l} ; \mathrm{n}=7$ ), 3,4-dihydro-5-[4-(1-piperidinyl)butoxy]-1(2H)- isoquinolinone (DPQ; 10 mg/kg; n=8) (14), or N-(1-naphthyl)ethylenediamine dihydrochloride $(1400 \mathrm{~W} ; 10 \mathrm{mg} / \mathrm{kg} ; \mathrm{n}=8)(17)$. Both DPQ and 1400W were dissolved in $100 \mu \mathrm{l}$ DMSO.

Measurement of cardiac function. The measurement was performed as previously described (18). At three days, and at two and four weeks following MI, the left ventricular (LV) dimension and function were assessed by 2-D transthoracic echocardiography on the isoflurane-anesthetized rats. A $12.5 \mathrm{mHz}$ linear-array probe was used, which has been specifically designed for rat cardiac ultrasonic studies, with an HP Sonos 7500 Imaging System (Philips Medical Systems, Andover, MA, USA). LV end-systolic dimension (ESD) and end-diastolic dimension (EDD) were measured from the short-axis view of the LV at the papillary muscle level. Fractional shortening (FS) was used as a sensitive marker of systolic function, and was calculated using the following equation: FS $(\%)=((E D D-E S D) / E D D) \times 100$. All of the measurements were averaged on three consecutive cardiac cycles, and analyzed by two independent researchers. The mice were humanely euthanized under anesthesia.

Terminal deoxynucleotidyl-transferase-mediated dUTP nick end labeling (TUNEL). Four weeks following MI, the rats were sacrificed and the hearts were harvested, fixed in $4 \%$ paraformaldehyde, embedded in paraffin, and cut into $5 \mu \mathrm{m}$ sections. A TUNEL assay was used to evaluate apoptotic activity. A commercially available apoptosis detection kit (Roche Diagnostics GmbH, Mannheim, Germany) was used. The terminal deoxynucleotidyl transferase (TdT) reaction was carried out for $1 \mathrm{~h}$ at $37^{\circ} \mathrm{C}$, and then 3,3'-diaminobenzidine (DAB) chromogen was applied to the samples. Hematoxylin was used as a counterstain. Myocardial apoptosis was assessed in the area at risk (AAR), as described previously (16). Briefly, in each section, the number of cardiomyocytes and the number of TUNEL-positive cardiomyocyte nuclei, were analyzed under a bright field microscope (Olympus Corporation, Tokyo, Japan). Image-Pro Plus v6.0 (Media Cybernetics, Inc., Rockville, MD, USA) was used to quantify the apoptotic activity, by randomly counting 10 fields of the section, at x400 magnification.

Caspase-3 activity assay. Caspase-3 activity was measured using a commercial kit, according to the manufacturer's instructions (Beyotime Institute of Biotechnology, Haimen, China). In the presence of caspase-3, acetyl-Asp-Glu-Val-Asp p-nitroanilide (Ac-DEVD-pNA) is hydrolysed to the yellow product, $\mathrm{p}$-nitroaniline(pNA). The concentration of $\mathrm{pNA}$ was measured at $405 \mathrm{~nm}$, using a spectrophotometer (Shanghai Optical Instrument Factory, Shanghai, China).

Western blot analysis. The proteins were extracted from the frozen cardiac tissue of each group using radioimmunoprecipitation assay lysis buffer (Beyotime Institute of Biotechnology). The protein samples were quantified with a BCA Protein Assay kit (Beyotime Institute of Biotechnology, Shanghai, China). Equal amounts of protein $(50 \mu \mathrm{g})$ were loaded onto $10 \%$ SDS-PAGE gels and separated by electrophoresis. The blots were then transferred to nitrocellulose membranes (Bio-Rad Laboratories, Inc., Hercules, CA, USA). The membranes were blocked at room temperature with $5 \%$ nonfat milk, in Tris-buffered saline containing $0.1 \%$ Tween $^{\circledR} 20$ (TBST), and then incubated at $4^{\circ} \mathrm{C}$ overnight with an appropriate primary antibody: Rabbit anti- $\beta$-actin (1:2,000 dilution; Santa Cruz Biotechnology, Dallas, TX, USA), rabbit anti-cleaved caspase-3 (1:1,000 dilution; Cell Signaling Technology, Danvers, MA, USA), rabbit anti-cleaved PARP (1:500 dilution; Abcam, Cambridge, MA, USA), or rabbit 
anti-iNOS (1:200 dilution; Santa Cruz Biotechnology). The membranes were then washed with TBST and incubated with horseradish peroxidase (HRP)-conjugated goat anti-rabbit secondary antibody (1:5,000 dilution; Jingmei Biotech Co., Ltd., Shenzhen, China) for $2 \mathrm{~h}$ at room temperature. Following a further three washes with TBST, the blots were visualized using Enhanced Chemiluminescence Plus reagents (EMD Millipore, Billerica, MA, USA).

Immunofluorescence. The heart tissues were fixed in $4 \%$ polyformaldehyde overnight and stored at $-4^{\circ} \mathrm{C}$. Serial cryosections (4-5 $\mu \mathrm{m}$ ) embedded in OCT compound were made by using a paraffin slicing machine. The serial cryosections $(6 \mu \mathrm{m})$ of the infarct-related area (IRA) were permeabilized in phosphate-buffered saline (PBS), containing 0.5\% Triton X-100. After being blocked with normal goat serum, the cryosections were incubated with the following primary antibodies, overnight at $4^{\circ} \mathrm{C}$ : Rabbit anti-PAR antibody (1:200 dilution; Santa Cruz Biotechnology), or rabbit anti-3NT (1:500 dilution; Abcam). The cryosections were then incubated with a HRP conjugated secondary antibody (1:5,000 dilution; Jingmei Biotech Co., Ltd., Shenzhen, China). As P3L2.. A small amount of Prolong Gold antifade reagent, with 4', 6-diamidino-2-phenylindole (DAPI; Invitrogen Life Technologies, Carlsbad, CA, USA) was used to seal the coverslips. The immunofluorescent images were captured using a P3L21 laser scanning confocal microscope (LSM710; Carl Zeiss AG, Oberchoken, Germany) and analyzed using Image Pro Plus 6.0 (Media Cybernetics, Inc., Rockville, MD, USA).

Superoxide anion $\left(\mathrm{O}_{2}^{-}\right)$production assay. Following treatment, the IRA of the cardiac tissues were incubated with a fluorescent probe of $\mathrm{O}_{2}{ }^{-}$, dihydroethidium (DHE), for $30 \mathrm{~min}$ at $37^{\circ} \mathrm{C}$. The IRA samples were washed with PBS, and the fluorescent signals were measured at $535 \mathrm{~nm}$, using a laser scanning confocal microscope. The fluorescence intensity was measured for $\geq 10$ fields per sample. Three independent experiments were performed.

Statistical analyses. All of the data are expressed as the means \pm standard deviation. SPSS for Windows version 16.0 (SPSS Inc, Chicago, IL, USA) was used for statistical analyses. Intergroup comparisons were performed using a two tailed student's t test or a one-way analysis of variance, followed by a test of least significant difference (when equal variances were assumed) or a Dunnett's T3 (when equal variances were not assumed). A P $<0.05$ was considered to indicate a statistically significant difference.

\section{Results}

Three rats in the MI group and two rats from both the DPQ and $1400 \mathrm{~W}$ groups succumbed to natural causes. Perioperative mortality $(<24$ hours following coronary ligation) ranged between $20-30 \%$, and was not affected by the surgery or the dose of the drugs. There was no mortality following the first 24 hours, up to the end of the experiment (4 weeks).

Inhibition of PARPI and iNOS activity improves cardiac function following MI. During the four weeks following coronary
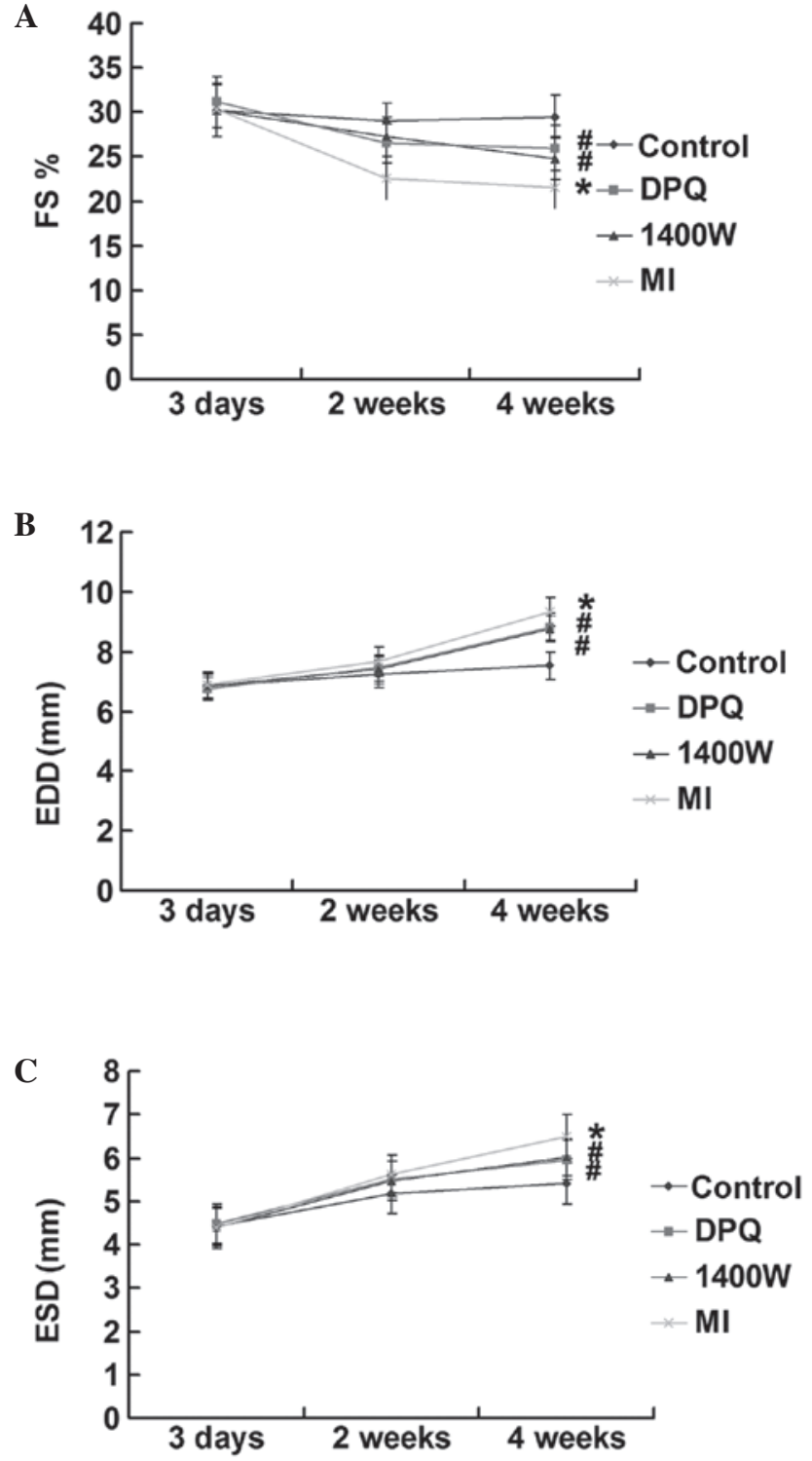

Figure 1. Evaluation of left ventricular dimension and function by echocardiography. (A) Fractional shortening (FS), (B) end-diastolic diameter (EDD), and (C) end-systolic diameter (ESD) of the four groups following myocardial infarction (MI). FS was decreased, and EDD and ESD were increased following MI, " $\mathrm{P}<0.05$ vs the control group; ${ }^{\text {}} \mathrm{P}<0.05$ vs the MI group. DPQ, 3,4-dihydro-5-[4-(1-piperidinyl)butoxy]-1(2H)- isoquinolinone; $1400 \mathrm{~W}$, $\mathrm{N}$-(1-naphthyl)ethylenediamine dihydrochloride.

ligation, the cardiac function of the rats was observed by serial echocardiography, and the FS, LV EDD and ESD were determined (Fig. 1A-C). The control group maintained consistent cardiac function, during the four weeks. In the MI group, the EDD and ESD increased by 19.30 and $16.65 \%$, respectively, four weeks after surgery, and the FS decreased by $26.87 \%$, as compared with the control group $(\mathrm{P}<0.05)$. The echo indices of FS and LV enlargement in the DPQ and $1400 \mathrm{~W}$ groups, following MI, were lower as compared with the control group; however, the reduction was less severe, as compared with the MI group ( $\mathrm{P}<0.05$; Fig. 1A-C).

Inhibition of PARPI and iNOS activity reduces MI-induced apoptosis. The rate of apoptosis was measured histologically by TUNEL assay (Fig. 2A). The average integral optical 
A

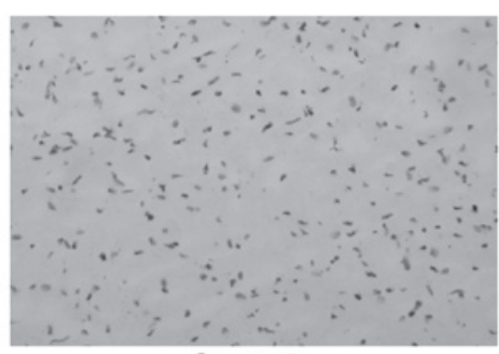

Control

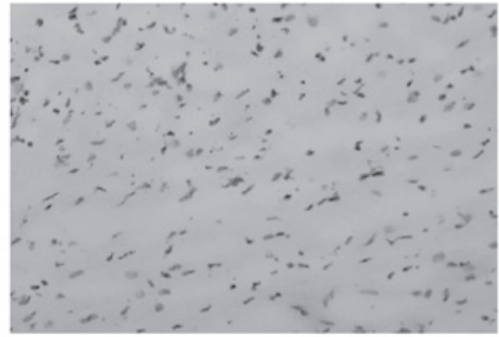

$\mathrm{DPQ}$

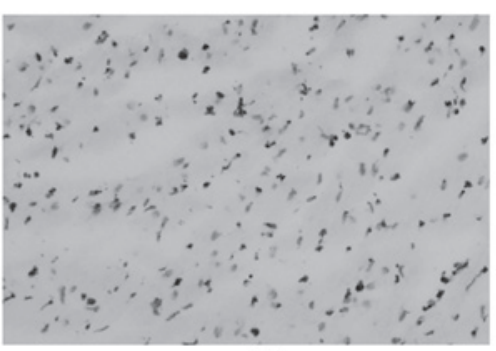

MI

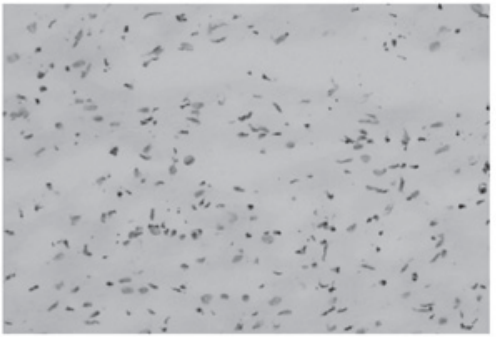

$1400 \mathrm{~W}$

B

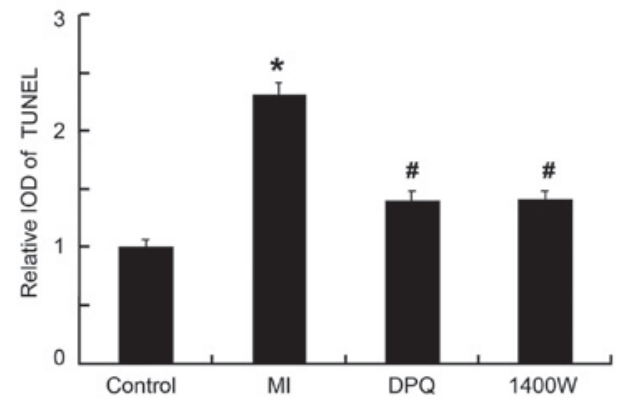

Figure 2. Terminal deoxynucleotidyl-transferase-mediated dUTP nick end labeling (TUNEL) positive cardiomyocytes in the myocardium, four weeks following myocardial infarction (MI). (A) Representative examples of TUNEL staining in myocardium (3,3'-diaminobenzidine staining; magnification, x400). (B) Percentage of TUNEL-positive cardiomyocytes in the myocardium. Control $(n=10)$; MI $(n=7)$; DPQ $(n=8) ; 1400 \mathrm{~W}(\mathrm{n}=8)$. " $\mathrm{P}<0.05$ vs the control group; ${ }^{\#} \mathrm{P}<0.05$ vs the MI group. DPQ, 3,4-dihydro-5-[4-(1-piperidinyl)butoxy]-1(2H)- isoquinolinone; 1400W, N-(1-naphthyl)ethylenediamine dihydrochloride; IOD, integral optical density.

A

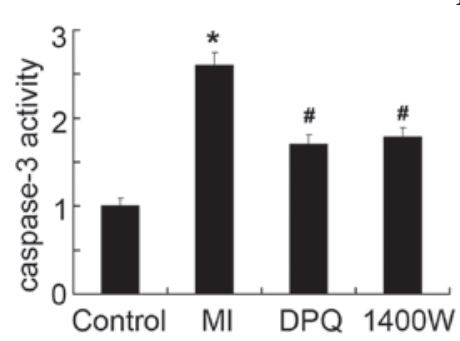

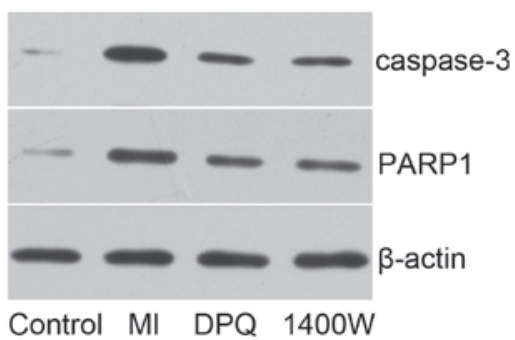

Control MI DPQ 1400W

C

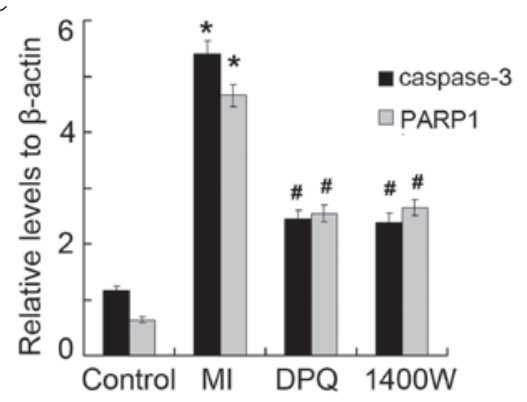

Figure 3. The protein expression levels of cleaved caspase-3 and cleaved poly (ADP-ribose) polymerase (PARP). (A) Caspase-3 activity of cardiomyocytes in the various groups. (B) The protein expression levels of cleaved caspase-3 and cleaved PARP, as determined by western blot analysis. (C) Quantitative analysis of cleaved caspase-3 and cleaved PARP, normalized to $\beta$-actin. " $\mathrm{P}<0.05$ vs the control group; ${ }^{*} \mathrm{P}<0.05$ vs the MI group. DPQ, 3,4-dihydro-5-[4-(1-piperidinyl) butoxy]-1(2H)- isoquinolinone; 1400W, N-(1-naphthyl)ethylenediamine dihydrochloride. MI, myocardial infarction. 
A
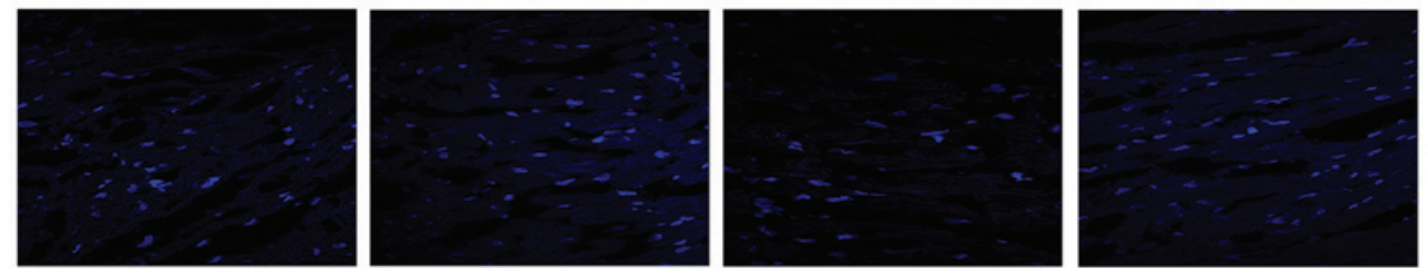

DAPI
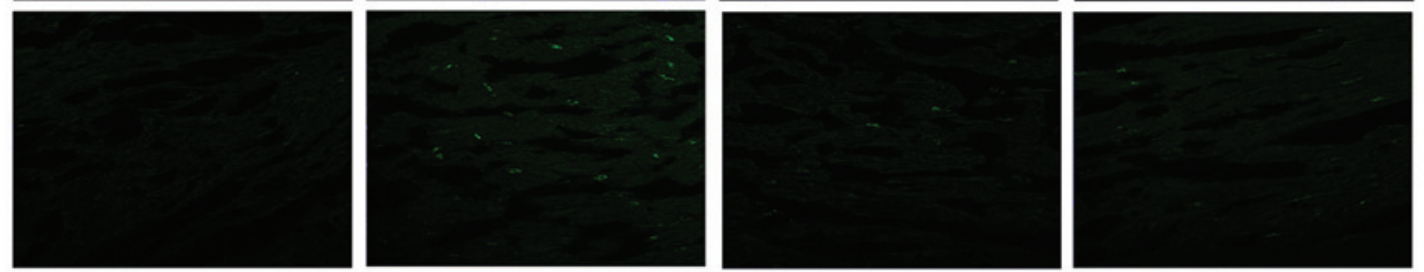

PAR
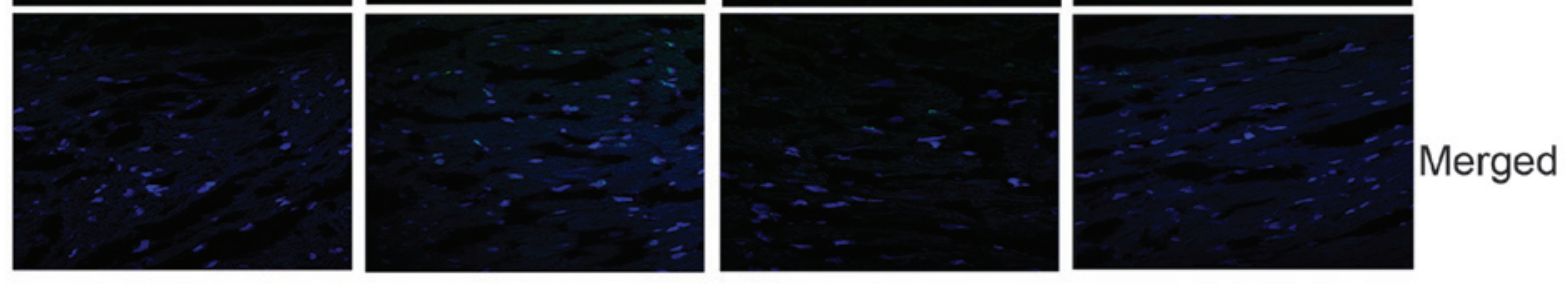

B

Control

$\mathrm{MI}$

$\mathrm{DPQ}$

1400W

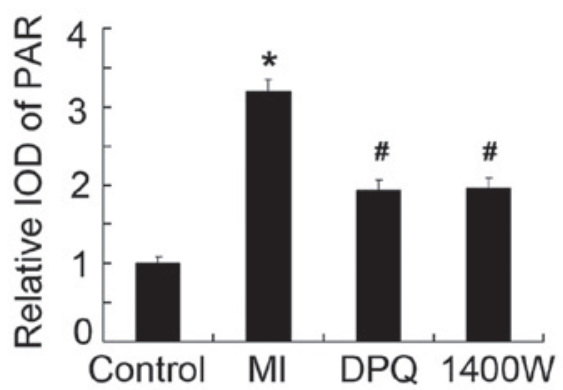

C

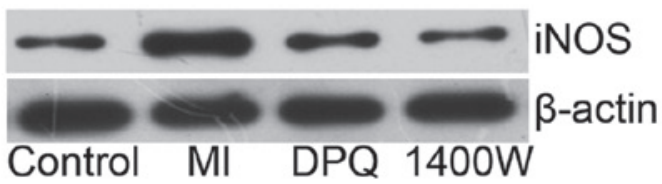

D

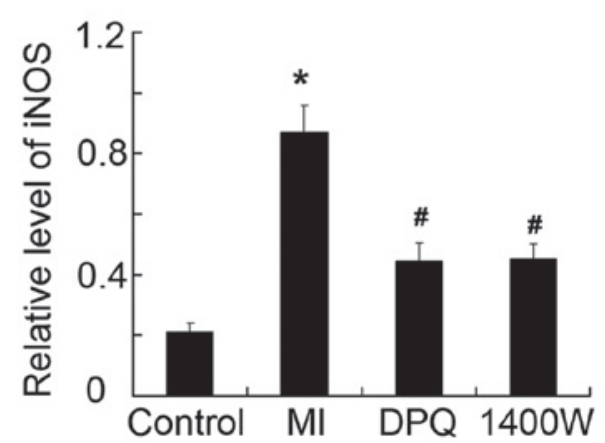

Figure 4. The protein expression levels of poly (ADP-ribose) (PAR) (active PAR polymerase), and inducible nitric oxide synthase (iNOS), in the myocardium of the various groups. (A) Representative fluorescent images of the myocardium, acquired by laser scanning confocal microscopy (magnification, $\mathrm{x} 400$ ); PAR staining is green, and 4', 6-diamidino-2-phenylindole (DAPI) staining for the cell nuclei is blue. (B) Quantitative analysis of PAR, expressed as a fold increase over the control group. (C) The protein expression levels of iNOS were examined by western blot analysis, with the indicated antibody. (D) Quantitative analysis of iNOS, relative to $\beta$-actin. Control, $\mathrm{n}=10$; MI, $\mathrm{n}=7$; DPQ, $\mathrm{n}=8 ; 1400 \mathrm{~W}, \mathrm{n}=8$. $\mathrm{P}<0.05$ vs the control group; \#P<0.05 vs the MI group. DPQ, 3,4-dihydro-5-[4-(1-piperidinyl)butoxy]-1(2H)- isoquinolinone; 1400W, N-(1-naphthyl)ethylenediamine dihydrochloride; IOD, integral optical density. MI, myocardial infarction.

density (IOD) for TUNEL-positive cardiomyocytes within the myocardial AAR was significantly increased in the MI group, as compared with the control group ( $4.05 \pm 0.41$ vs $1.75 \pm 0.12$, $\mathrm{P}<0.05$; Fig. 2B). The rate of apoptosis was significantly reduced, by 39.71 and by $39.00 \%$ in the DPQ and $1400 \mathrm{~W}$ treatment groups, respectively, as compared with the MI group ( $\mathrm{P}<0.05$, Fig. 2B). There were no significant differences between the rate of apoptosis in the DPQ and $1400 \mathrm{~W}$ groups.

Inhibition of PARPI and INOS attenuates apoptosis in the $A A R$ by regulating the expression levels of cleaved caspase- 3 and PARPI. The main functions of caspase- 3 and PARP1 are 
A
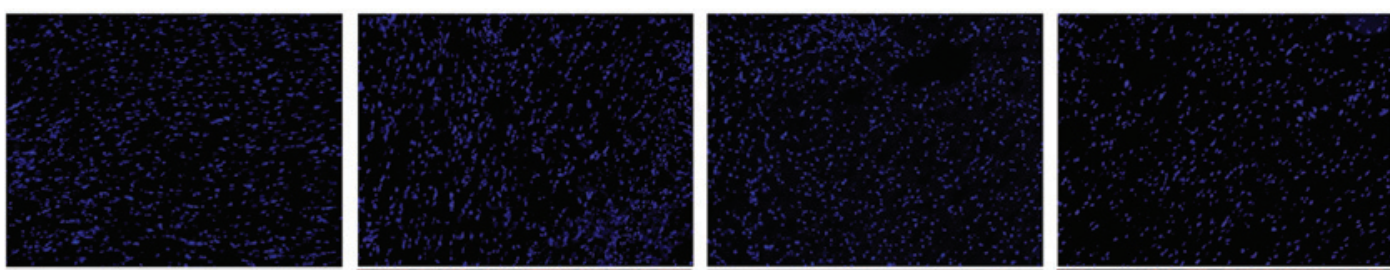

DAPI
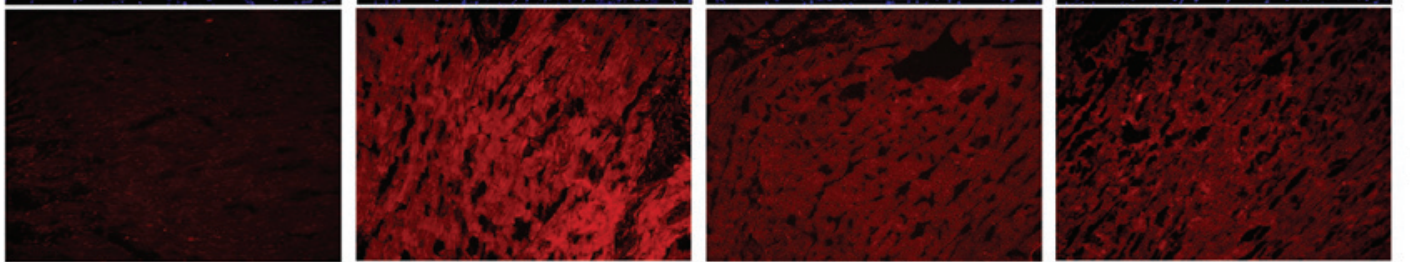

DHE

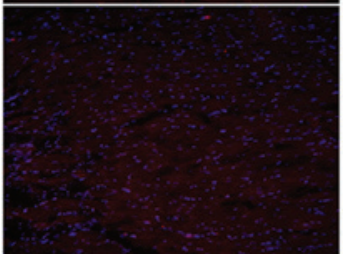

Control

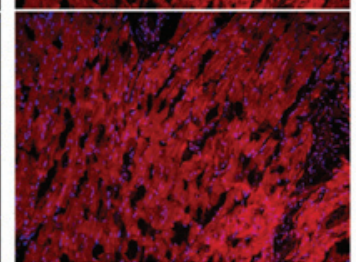

MI

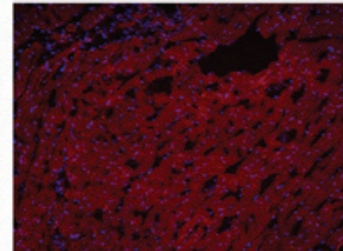

DPQ

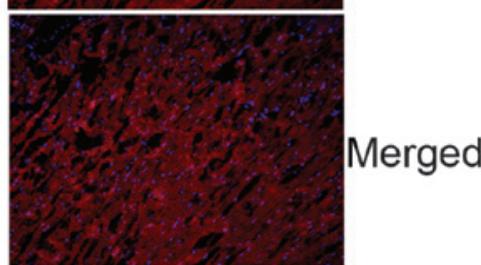

1400W

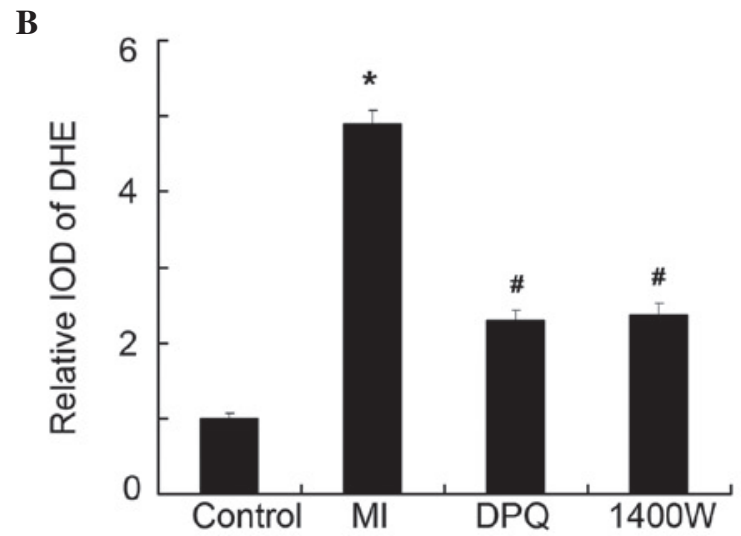

Figure 5. The expression of $\mathrm{O}_{2}^{-}$detected by dihydroethidium (DHE). (A) The representative images of $\mathrm{O}_{2}^{-}$detected by DHE (red) in the myocardium, 4', 6-diamidino-2-phenylindole (DAPI) staining for the cell nuclei is blue. (B) Quantitative analysis of DHE, expressed as a fold increase over the control group. Control, $\mathrm{n}=10 ; \mathrm{MI}, \mathrm{n}=7$; DPQ, $\mathrm{n}=8 ; 1400 \mathrm{~W}, \mathrm{n}=8$. ${ }^{*} \mathrm{P}<0.05$ vs the control group; ${ }^{*} \mathrm{P}<0.05$ vs the MI group. DPQ, 3,4-dihydro-5-[4-(1-piperidinyl)butoxy]-1(2H)isoquinolinone; 1400W, N-(1-naphthyl)ethylenediamine dihydrochloride; IOD, integral optical density. MI, myocardial infarction.

in apoptosis. The protein expression levels of caspase- 3 and PARP1 were examined (Fig. 3). The activity of caspase-3 was 2.60-fold higher in the MI group, as compared with the control group $(\mathrm{P}<0.05$, Fig. 3A). Treatment with DPQ or $1400 \mathrm{~W}$ significantly decreased caspase-3 activity, as compared with the MI group ( $\mathrm{P}<0.05$, Fig. 3A). There was no difference in caspase-3 activity between the two treatment groups. Similar results were determined by western blot analysis (Fig. 3B), and the enhancement of cleaved caspase-3 and PARP1 induced by MI was attenuated by treatment with DPQ or 1400W ( $\mathrm{P}<0.05$; Fig. 3C). These results were concordant with the rate of apoptosis detected in each of the groups.

$D P Q$ and 1400W effectively inhibit MI-induced PARP1 and $i N O S$ activity. In order to verify the respective inhibition efficiency of DPQ and 1400W on PARP1 and iNOS, the expression levels of PAR (active PARP) and iNOS were detected in the myocardium of the various groups (Fig. 4). The activity of PAR was detected by immunofluorescence (Fig. 4A). The average IOD was enhanced 3.20-fold in the MI group, as compared with the control $(\mathrm{P}<0.05$, Fig. 4B); however, treatment with DPQ or $1400 \mathrm{~W}$ reduced MI-induced PAR activity by 39.38 and $38.75 \%$, respectively ( $\mathrm{P}<0.05$; Fig. 4B). Simultaneously, the expression levels of iNOS were enhanced 4.14-fold following MI, as compared with the control group, as determined by western blotting $(\mathrm{P}<0.05$, Fig. $4 \mathrm{C}$ and $\mathrm{D})$. The inhibition efficiency of DPQ and 1400W was 51.14 and $51.84 \%$ respectively, as compared with the $\mathrm{MI}$ group $(\mathrm{P}<0.05$, Fig. $4 \mathrm{C}$ and D). Notably, DPQ and $1400 \mathrm{~W}$ could interact with each other in the inhibition of PARP1 and iNOS.

Inhibition of PARP1 and $i N O S$ repressed $\mathrm{O}_{2}^{-}$and nitrotyrosine (3-NT), induced by $M I$. To further elucidate the signaling pathways involved in MI-induced apoptosis, the expression of $\mathrm{O}_{2}{ }^{-}$and the production of $\mathrm{NO}(3-\mathrm{NT})$ was determined. The production of intracellular $\mathrm{O}_{2}^{-}$was significantly increased in 
A
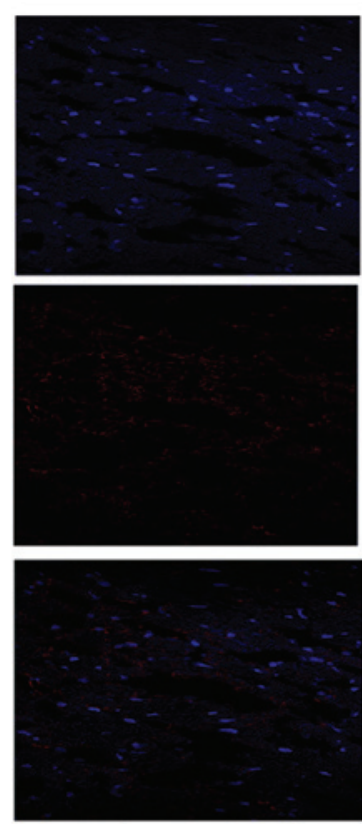

Control
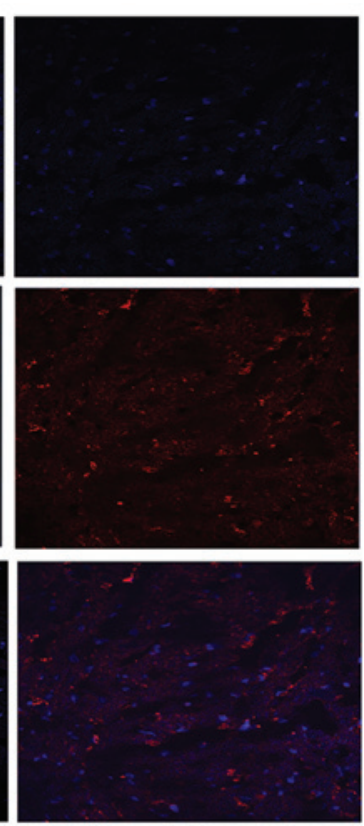

MI
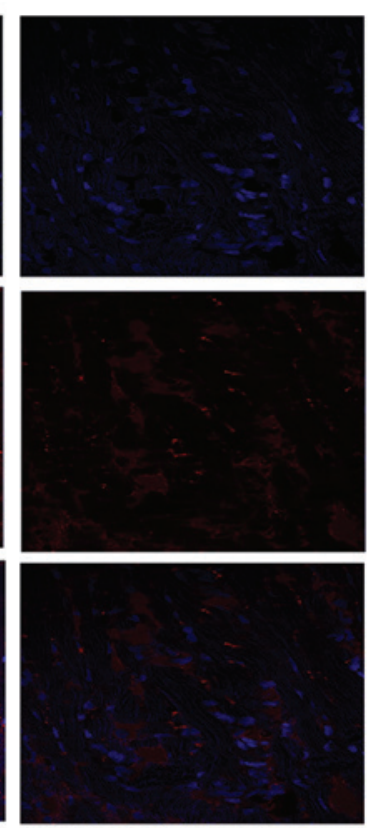

DPQ
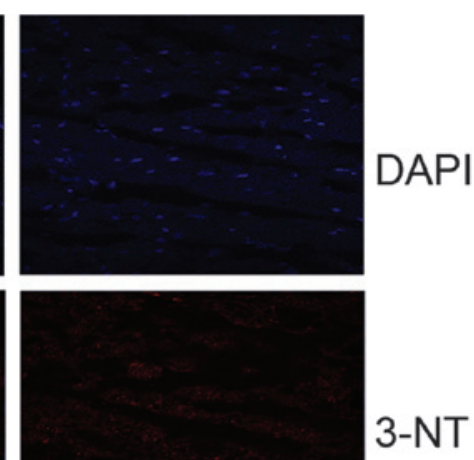

3-NT
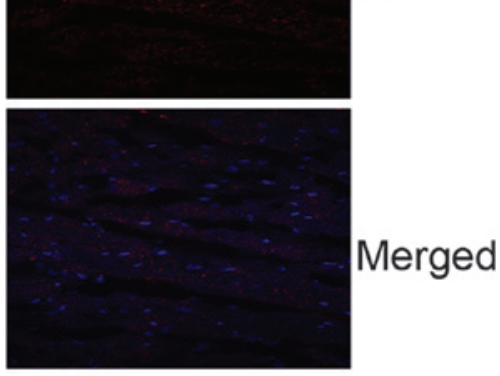

1400W

B

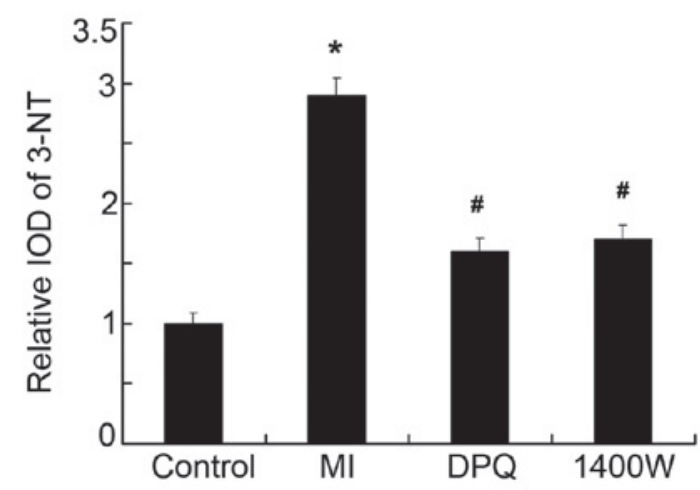

Figure 6. The relative levels of nitrotyrosine (3-NT) in the myocardium. (A) Fluorescent micrographs showing the relative level of 3-NT (red) in the myocardium, 4', 6-diamidino-2-phenylindole (DAPI)staining for the cell nuclei is blue. (B) Quantitative analysis of 3-NT, expressed as a fold increase over the control group. Control, $\mathrm{n}=10 ; \mathrm{MI}, \mathrm{n}=7$; DPQ, $\mathrm{n}=8 ; 1400 \mathrm{~W}, \mathrm{n}=8 .{ }^{*} \mathrm{P}<0.05$ vs the control group; ${ }^{*} \mathrm{P}<0.05$ vs the MI group. DPQ, 3,4-dihydro-5-[4-(1-piperidinyl)butoxy]-1(2H)- isoquinolinone; 1400W, N-(1-naphthyl)ethylenediamine dihydrochloride; IOD, integral optical density. MI, myocardial infarction.

the MI group, as compared with the control group, as determined by DHE detection $(\mathrm{P}<0.05$, Fig. $5 \mathrm{~A}$ and B). Similarly, the expression of 3-NT was significantly higher in the MI group, as compared with the control group, as determined by immunofluorescence $(\mathrm{P}<0.05$, Figure $6 \mathrm{~A}, \mathrm{~B})$. Treatment with DPQ or $1400 \mathrm{~W}$ effectively reversed the high $\mathrm{O}_{2}^{-}$levels induced by MI, by 53.07 and $51.43 \%(<0.05$, Fig. 5A and B), and reduced $3-\mathrm{NT}$ expression by 44.87 and $41.38 \%(\mathrm{P}<0.05$, Fig. 6A and B), respectively.

\section{Discussion}

The present study tested the hypothesis that PARP1 and iNOS contribute to the deleterious effects on cardiac function, exerted by cardiomyocyte apoptosis, following MI. PARP1 and iNOS were shown to be upregulated, alongside an increased rate of caspase-3-dependent apoptosis, and deterioration of cardiac function in rats, following an MI. The PARP inhibitor
DPQ and the iNOS inhibitor $1400 \mathrm{~W}$ attenuated these effects. Furthermore, the reduction in the rate of apoptosis, by DPQ and $1400 \mathrm{~W}$, was associated with the reduced production of $\mathrm{O}_{2}{ }^{-}$and 3-NT in the ischemic myocardium.

Previous studies have evaluated cardiac function following induction of MI by serial echocardiography $(2,19)$, and demonstrated that it is associated with apoptosis and inflammation in the AAR (20). In concordance with previous reports, the results of the present study showed that apoptosis following MI and production of iNOS were markedly elevated, with deterioration of cardiac function. Considering the involvement of PARP1 in various cardiovascular and inflammatory diseases, these results emphasize its role in the pathogenesis of MI.

Previous evidence has suggested that PARP may be excessively activated by reactive oxygen and nitrogen species in cardiomyocytes and endothelial cells, during myocardial ischemia/reperfusion injury, various forms of heart failure or 
cardiomyopathies, circulatory shock, cardiovascular aging, diabetic complications, myocardial hypertrophy, atherosclerosis, and vascular remodeling following injury (21). Furthermore, severe DNA damage may lead to initiation of the second apoptotic pathway, in which caspases inactivate PARP1, by cleaving it into two fragments, therefore preventing it from responding to DNA strand breaks $(22,23)$. Reactive oxygen species, which have been implicated in cardiac pathophysiology, can trigger apoptosis of myocytes by upregulating pro-apoptotic proteins, such as Bcl-2-associated X protein and caspases, and the mitochondria-dependent pathway (24). The results of the present study indicated that inhibition of PARP1 with DPQ, could reduce $\mathrm{O}_{2}^{-}$and $\mathrm{ONOO}^{-}$in rats following MI, and reduce caspase-3-dependent apoptosis. These results were concordant with previous findings that the PARP1 inhibitor PJ34 limited myocardial damage, due to post-ischemic reperfusion, by decreasing $\mathrm{NAD}^{+}$and $\mathrm{ATP}$, and the caspase-dependent pathway (25).

Another role of PARP1 is its regulation of inflammation at the transcriptional level (e.g., iNOS, intracellular adhesion molecule-1, cyclooxygenase-2). The absence of functional PARP-1 has been shown to decrease the expression levels of proinflammatory mediators, including cytokines, chemokines, adhesion molecules and enzymes, such as iNOS (26). Previous results have suggested that iNOS expression contributes to PARP activation in cerebral ischemia, and a previously unrecognized deleterious interaction between iNOS and PARP has been identified (27). The present study demonstrated that inhibition of PARP1 reduced the expression of iNOS, meanwhile the inhibitor of iNOS, $1400 \mathrm{~W}$, attenuated the expression of PARP1 and resulted in the reduction of apoptosis. The interaction between PARP1 and iNOS requires further study.

iNOS is one of the most important NO donors, and is associated with numerous important pathophysiological processes in various conditions, including MI and heart failure $(28,29)$. Cell apoptosis and NT formation have previously been attenuated by selective iNOS inhibitors, or in iNOS knockout mice (30). Peroxynitrite is a reactive oxidant which is formed by reaction of $\mathrm{NO}$ with the superoxide anion (11). $\mathrm{ONOO}^{-}$has been shown to function as an important trigger of myocardial necrosis and apoptosis in various cardiac pathologies, by inducing oxidative DNA damage, which may lead to the activation of the DNA repair enzyme PARP. PARP subsequently consumes cellular $\mathrm{NAD}^{+}$, ultimately leading to ATP depletion and necrotic cell death $(21,31,32)$. The results of the present study showed that the iNOS inhibitor, $1400 \mathrm{~W}$, could attenuate NT formation and apoptosis, and could reduce the activity of PARP1.

In conclusion, the present study provided novel evidence that pharmacological inhibition of PARP1 with DPQ and iNOS with $1400 \mathrm{~W}$, improved cardiac function in rats, following MI, by attenuating cell apoptosis and inflammation. The predicted pathway of these effects is that DPQ may downregulate the inflammatory factor iNOS and its effects on $\mathrm{O}_{2}{ }^{-}$and $\mathrm{ONOO}^{-}$in $\mathrm{MI}$ rats, and reduce the rate of caspase-3-dependent apoptosis. In addition, inhibition of iNOS decreased the levels of reactive oxygen and nitrogen species, resulting in the reduction of DNA single strand breaks and PARP1 activiation, leading to similar protection in ischemic myocardium. The effects of PARP1 and iNOS inhibitors may be exploited for the experimental therapy of disease.

\section{Acknowledgements}

The present study was supported by the Seed Fund of The Second Hospital of Shandong University (no. S2013010010).

\section{References}

1. Ozaki K, Sato H, Inoue K, et al: SNPs in BRAP associated with risk of myocardial infarction in Asian populations. Nat Genet 41: 329-333, 2009.

2. Chacko SM, Khan M, Kuppusamy ML, et al: Myocardial oxygenation and functional recovery in infarct rat hearts transplanted with mesenchymal stem cells. Am J Physiol Heart Circ Physiol 296: H1263-H1273, 2009.

3. Cohen-Armon M: PARP-1 activation in the ERK signaling pathway. Trends Pharmacol Sci 28: 556-560, 2007.

4. Hassa PO, Haenni SS, Elser M and Hottiger MO: Nuclear ADP-ribosylation reactions in mammalian cells: where are we today and where are we going? Microbiol Mol Biol Rev 70: 789-829, 2006

5. Szabó C, Zingarelli B, O'Connor $\mathrm{M}$ and Salzman AL: DNA strand breakage, activation of poly (ADP-ribose) synthetase, and cellular energy depletion are involved in the cytotoxicity of macrophages and smooth muscle cells exposed to peroxynitrite. Proc Natl Acad Sci USA 93: 1753-1758, 1996.

6. Ullrich O, Diestel A, Eyüpoglu IY and Nitsch R: Regulation of microglial expression of integrins by poly(ADP-ribose) polymerase-1. Nat Cell Biol 3: 1035-1042, 2001.

7. Sharp C, Warren A, Oshima T, Williams L, Li JH and Alexander JS: Poly ADP ribose-polymerase inhibitors prevent the upregulation of ICAM-1 and E-selectin in response to Th1 cytokine stimulation. Inflammation 25: 157-163, 2001.

8. Hassa PO and Hottiger MO: The functional role of poly (ADP-ribose)polymerase 1 as novel coactivator of NF-kappaB in inflammatory disorders. Cell Mol Life Sci 59: 1534-1553, 2002.

9. Carrillo A, Monreal Y, Ramírez P, et al: Transcription regulation of TNF-alpha-early response genes by poly(ADP-ribose) polymerase-1 in murine heart endothelial cells. Nucleic Acids Res 32: 757-766, 2004.

10. Szabó C, Zanchi A, Komjáti K, et al: Poly(ADP-Ribose) polymerase is activated in subjects at risk of developing type 2 diabetes and is associated with impaired vascular reactivity. Circulation 106: 2680-2686, 2002.

11. Pacher P, Beckman JS and Liaudet L: Nitric oxide and peroxynitrite in health and disease. Physiol Rev 87: 315-424, 2007.

12. Pacher P and Szabó C: Role of poly(ADP-ribose) polymerase-1 activation in the pathogenesis of diabetic complications: endothelial dysfunction, as a common underlying theme. Antioxid Redox Signal 7: 1568-1580, 2005.

13. Martinet W, Knaapen MW, De Meyer GR, Herman AG and Kockx MM: Elevated levels of oxidative DNA damage and DNA repair enzymes in human atherosclerotic plaques. Circulation 106: 927-932, 2002.

14. Szabó C: Pharmacological inhibition of poly(ADP-ribose) polymerase in cardiovascular disorders: future directions. Curr Vasc Pharmacol 3: 301-303, 2005.

15. Szabó C, Ischiropoulos Hand RadiR: Peroxynitrite: biochemistry, pathophysiology and development of therapeutics. Nat Rev Drug Discov 6: 662-680, 2007.

16. Ahmet I, Lakatta EG and Talan MI: Pharmacological stimulation of beta2-adrenergic receptors (beta2AR) enhances therapeutic effectiveness of betalAR blockade in rodent dilated ischemic cardiomyopathy. Heart Fail Rev 10: 289-296, 2005.

17. Matsuhisa S, Otani H, Okazaki T, et al: Angiotensin II type 1 receptor blocker preserves tolerance to ischemia-reperfusion injury in Dahl salt-sensitive rat heart. Am J Physiol Heart Circ Physiol 294: H2473-H2479, 2008.

18. Wang Y, Zhang G, Hou Y, et al: Transplantation of microencapsulated Schwann cells and mesenchymal stem cells augment angiogenesis and improve heart function. Mol Cell Biochem 366: $139-147,2012$

19. Khan M, Meduru S, Mohan IK, et al: Hyperbaric oxygenation enhances transplanted cell graft and functional recovery in the infarct heart. J Mol Cell Cardiol 47: 275-287, 2009. 
20. Ahmet I, Tae HJ, Juhaszova M, et al: A small nonerythropoietic helix B surface peptide based upon erythropoietin structure is cardioprotective against ischemic myocardial damage. Mol Med 17: 194-200, 2011.

21. Pacher P and Szabó C: Role of poly(ADP-ribose) polymerase 1 (PARP-1) in cardiovascular diseases: the therapeutic potential of PARP inhibitors. Cardiovasc Drug Rev 25: 235-260, 2007.

22. Levrand S, Vannay-Bouchiche C, Pesse B, et al: Peroxynitrite is a major trigger of cardiomyocyte apoptosis in vitro and in vivo. Free Radic Biol Med 41: 886-895, 2006.

23. Virag L and Szabó C: The therapeutic potential of poly(ADP-ribose) polymerase inhibitors. Pharmacol Rev 54: $375-429,2002$

24. Kumar D and Jugdutt BI: Apoptosis and oxidants in the heart. J Lab Clin Med 142: 288-297, 2003.

25. Fiorillo C, Ponziani V, Giannini L, et al: Protective effects of the PARP-1 inhibitor PJ34 in hypoxic-reoxygenated cardiomyoblasts. Cell Mol Life Sci 63: 3061-3071, 2006.

26. Szabó C: Poly(ADP-ribose) polymerase activation by reactive nitrogen species - relevance for the pathogenesis of inflammation. Nitric Oxide 14: 169-179, 2006.
27. Sarkar D and Fisher PB: Molecular mechanisms of aging-associated inflammation. Cancer Lett 236: 13-23, 2006.

28. Gilson WD, Epstein FH, Yang Z, et al: Borderzone contractile dysfunction is transiently attenuated and left ventricular structural remodeling is markedly reduced following reperfused myocardial infarction in inducible nitric oxide synthase knockout mice. J Am Coll Cardiol 50: 1799-807, 2007.

29. Liu YH, Carretero OA, Cingolani OH, et al: Role of inducible nitric oxide synthase in cardiac function and remodeling in mice with heart failure due to myocardial infarction. Am J Physiol Heart Circ Physiol 289: H2616-23, 2005.

30. Mukhopadhyay P, Rajesh M, Bátkai S, et al: Role of superoxide, nitric oxide, and peroxynitrite in doxorubicin-induced cell death in vivo and in vitro. Am J Physiol Heart Circ Physiol 296: H1466-H1483, 2009.

31. van Wijk SJ and Hageman GJ: Poly(ADP-ribose) polymerase-1 mediated caspase-independent cell death after ischemia/reperfusion. Free Radic Biol Med 39: 81-90, 2005

32. Zhang Y, Zhang X, Park TS and Gidday JM: Cerebral endothelial cell apoptosis after ischemia-reperfusion: role of PARP activation and AIF translocation. J Cereb Blood Flow Metab 25: 868-877, 2005. 